\title{
RELATIONS PONDÉRALES ENTRE L' ENCÉPHALE ET LE CORPS CHEZ LES CHIROPTÈRES
}

\author{
II. Espèces paléo-tropicales et paléarctiques. Discussion et conclusions
}

\author{
par \\ PAUL PIRLOT \\ Département des Sciences Biologiques, Université de Montréal, Montréal, Canada
}

\begin{abstract}
SUMMARY
The ratio brain weight/body weight is studied in $\mathbf{3 0}$ species of bats from Asia, Africa and Europe, in comparison with 20 other species from South America dealt with in a previous paper. The simple encephalic ratios, expressed in o/oo, vary considerably and about between the same limits on the four continents. Further, logarithmic regression lines (brain to body), when fitted to the representatives of various dietary types, provide slope and ordinate values that are visually distinguishable. Besides, the encephalic ratios themselves show, among bats, a negative correlation with the abolute somatic weight, as one might have expected. But, if one compares graphically, from that viewpoint, the Chiroptera on the one hand with a number of Insectivores and Primates on the other hand, one can observe that the first order is situated in an intermediary zone between the other two. A number of encephalic ratios for insect-eating bats are nevertheless intermingled with those for Insectivores; finally, some of the bats show clearly higher ratio-values than the others and those bats belong to ethologically specialized species (always from the viewpoint of the diet). Similarly high values of the encephalic ratio are a.so found amoung Primates, especially in man. This leads to the hypothesis that, at the origin of certain phyletic branchings, an increase of the encephalic nervous mass may take place (perhaps before any other size progress can occur). Such an increase would immediately constitute a selective advantage. However, the problem cannot be reduced to a mere increase in weight of the total brain until the details of cerebral structures have been studied in various groups so that the evolution of each part of the brain, in relation with particular needs for sensory controls, is better known.
\end{abstract}

Dans la première partie de ce travail (Pirlot, 1969a), j'ai présenté des données sur le rapport encéphale/corps chez 20 espèces de chiroptères néo-tropicaux. J'y ai montré qu'il existe une certaine correspondance positive entre le poids relatif moyen de l'encéphale et le groupe alimentaire auquel appartient chaque espèce. J'y ai souligné également la corrélation négative entre le rapport encéphale/corps et le poids absolu du corps. Toutefois, la discussion et les conclusions générales de cette étude ont été reportées logiquement jusqu'après l'examen d'espèces de l'Ancien Monde.

\section{MATERIEL ET METHODE}

En 1967, j'ai pu récolter d'autres espèces de chauves-souris en Inde, en Malaisie (Selangor), dans l'Ile de Bornéo (Sarawak) et au Japon. Ce matériel, destiné à des recherches de neuro-anatomie, a fourni accessoirement de nouvelles données numériques sur la relation entre le poids du cerveau et le poids du corps. La récolte totale en Asie m'a procuré 21 genres comprenant probablement 29 espèces. En raison du but principal de ces récoltes, je n'ai pas pu, dans la plupart des cas, peser les encéphales non fixés. J'ai pu toutefois obtenir en campagne les poids somatiques et encéphaliques à l'état frais de 46 spécimens appartenant aux quatre espèces suivantes:

\section{Famille Pteropidae - Cynopterus horsfieldii \\ - Cynopterus brachyotis \\ - Eonycteris spelaea}

Famille Hipposideridae - Hipposideros bicolor

D'autre part, grâce à l'amabilité du Dr. Heinz Stephan, du Max-Planck-Institut für Hirnforschung, de Francfort/Main, j'ai pu utiliser des données inédites obtenues sur 18 autres espèces et j'y ai ajouté des valeurs tirées de Dubois (1914), de Warncke (1908) et de Mangold-Wirz (1966) sur 8 autres espèces, toutes ces formes provenant de l'Ancien Monde. Voici la liste des 26 espèces pour lesquelles les quatre auteurs cités ont fourni des données pondérales. 
Famille Pteropidae

Famille Rhinolophidae

Famille Vespertilionidae

Sous-famille Vespertilioninae - Pipistrellus sp.

- Nyctalus noctula

- Myotis bocagei

- Myotis myotis

- Myotis daubentonii

- Myotis dasycmene

- Myotis bechsteinii

- Myotis nattereri

- Myotis mystacinus

- Vespertilio murinus

- Vespertilio serotinus

- Scotophilus gigas

Sous-famille Miniopterinae - Miniopterus

- Mormopterus (Tadarida) albiventer

- Chaerophon

leucostigma

Famille Nycteridae

Famille Hipposideridae
- Chaerophon limbatus

- Nycteris arge

- Hipposideros caffer rences biométriques entre des groupes de niveaux taxonomiques élevés.

\section{RESULTATS BRUTS}

Les 127 mesures obtenues sont présentées dans le graphique fig. 1, à l'exception de celles des plus grands ptéropidés qui exigeaient trop d'espace. Dans la table I sont indiqués les rapports encéphale/corps en $\%$ oo pour chaque espèce, $x$ indiquant toujours le poids du corps, y celui de l'encéphale

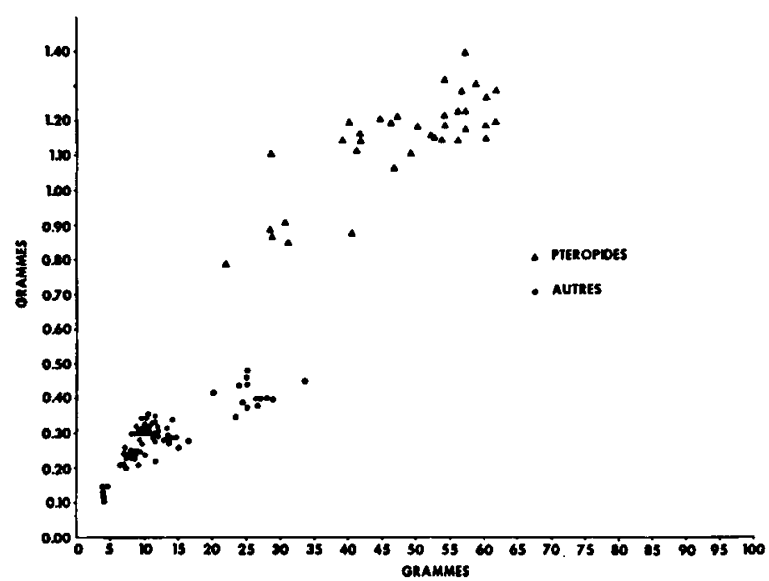

Fig. 1. Chiroptères de l'Ancien Monde. En ordonnées : poids encéphaliques. En abscisse : poids somatiques.

et $\mathrm{n}$ le nombre de spécimens utilisés; le poids somatique est entendu net, c'est-à-dire poids encéphalique déduit. Comme dans la première partie du travail, je n'ai utilisé que des spécimens apparemment adultes.

Ainsi exprimé, le poids relatif de l'encéphale est comparable, pour l'ensemble des espèces de la table I, à ce qu'il est chez les chauves-souris d'Amérique du Sud. Parmi les ptéropidés, c'est la forme la plus légère (Cynopterus brachyotis) qui possède l'encéphale le plus lourd relativement et, inversement, l'espèce la plus lourde (Pteropus edulis) possède le cerveau relativement le plus léger. Parmi les entomophages, la forme la plus légère est le vespertilioniné Myotis mystacinus qui présente le poids encéphalique relatif le plus élevé. Ceci confirme donc la corrélation négative d'ensemble sinon de détail entre poids relatif du cerveau et poids du corps chez les chiroptères Pirlot, 1969, que je commenterai plus loin. Quant à l'intérêt, en général, du poids encéphalique exprimé comme pourcentage (ou $\%$ ) du poids somatique, j'en discuterai prochainement dans un 
Table I. Rapports encéphale/corps chez certains chiroptères $\mathrm{x}=$ poids somatique moyen; $\mathrm{y} / \mathrm{x}=$ rapport $\mathrm{du}$ poids encéphalique moyen au poids somatique moyen; n $=$ nombre de spécimens utilisés

Spécimens d'Asie

(Coll. Pirlot)

Cynoglossus horsfieldii

$x \quad y / x$ en $\%$ n

C. brachyotis

Eonycteris spelaea

Hipposideros bicolor

\begin{tabular}{rrr}
52.80 & 23.1 & 16 \\
27.98 & 30.8 & 5 \\
48.48 & 24.1 & 14 \\
8.43 & 28.6 & 11 \\
\hline
\end{tabular}

Total 46

Spécimens d'Europe et d'Afrique

(Coll. Stephan)

Rousettus aegyptiacus

Casinycteris argynnis

130.0

Hysignathus monstruosus

Hipposideros caffer

Nycteris arge

Rhinolophus hipposideros A

Rhinolophus hipposideros B

Myotis bocagei

M. myotis

M. daubentonii

$M$. dasycmene

M. bechsteinii

$M$. nattereri

M. mystacinus

Pipistrellus sp.

Miniopterus schreibersi

Chaerophon leucostigma

C. limbatus

Mormopterus (Tadarida) albiventer 11.3
39.7

335.4

10.3

10.2

9.4

4.6

7.2

24.0

6.8

14.7

7.3

6.8

3.9

4.2

12.2

26.1

13.7 centre de gravité, un groupe plus ou moins dispersé allométriquement. Mais ceci n'offre, en pratique, aucun inconvénient sérieux lorsque les mensurations ont été fixées sur des spécimens qui étaient tous adultes et dont l'ensemble n'est pas affecté d'une très forte allométrie, ce qui est ici le cas, sauf pour une des espèces. Par elle-même et comme on le verra ci-après, cette simple formule met en évidence des problèmes fondamentaux que des procédés plus élaborés ne découvrent pas aussi clairement. Il est évident qu'il faut é g a l e m e n t recourir aux équations d'allométrie habituelles et je l'ai fait ici; mais ces équations n'ont pas que des avantages.

\section{COEFFICIENTS DE CORRELATION ET DE REGRESSION}

D'après l'équation logarithmique courante $\mathrm{Y}=\mathrm{B}+\mathrm{kX}$ où $\mathrm{Y}=\log \mathrm{y}, \mathrm{B}=\log \mathrm{b}$ et $\mathrm{X}=\log \mathrm{x}$ de la formule bien connue $y=b x^{k}$, on obtient, pour les relations intraspécifiques, les estimations de corrélation et de régression de la table II.

Les échantillons sont très petits et les corrélations pour les espèces séparées ne sont pas significatives $(P>0.05)$ ou ne le sont que douteusement (Hipposideros caffer). Les régressions ont également des valeurs très faibles; pour Myotis myotis et Chaerophon limbatus, celles-ci sont en outre négatives, ce qui ne présente aucun intérêt vu la petitesse des échantillons.

$\mathrm{Si}$ l'on groupe d'une part tous les frugivores (ptéropidés) et d'autre part tous les entomophages,

Spécimens de l'Ancien Monde

(Dubois, Warncke, Mangold-Wirz)

Pteropus edulis 120

P. edwarsi 325

8.4

22.1

Epomophorus gambianus

14.0

Vespertilio serotinus

305

23.1

13.4

$V$. murinus

13.7

15.1

23.2

10.5

Scotophilus gigas
Nyctalus noctula

14.4

Total 12

Total général 127

travail séparé. L'utilisation de ce rapport a été diversement commentée par les auteurs dans le passé. Il est évident que ce quotient encéphale/ corps ou $y / x$ ne constitue qu'une formule approchée puisqu'il est le rapport de la moy e n ne des mesures d'un organe à la mo y e n $\mathrm{n}$ e de celles du corps et que ce rapport varie avec la grandeur absolue de ce dernier. Il représente, en le concentrant en quelque sorte à son on obtient les coefficients de la table III. A celle-ci correspond le graphique fig. 2 dans lequel apparaissent, à côté des droites de régression des ptéropidés et des entomophages de l'Ancien Monde, celles des chiroptères néo-tropicaux reprises de la première partie de ce travail (Pirlot, 1969a). Nous y reviendrons dans la discussion.

\section{DISCUSSION ET CONCLUSIONS}

La discussion qui suit porte sur les données des deux parties de ce travail, c'est-à-dire que les chiroptères néo-tropicaux, paléo-tropicaux et paléarctiques y seront considérés ensemble.

A. Poids relatif de l'encéphale chez les chiroptères, les insectivores et les primates.

Il est intéressant de reprendre comparativement les données présentées sur les chauves-souris dans les deux parties de cette étude et celles publiées par divers auteurs sur d'autres mammifères. Je 


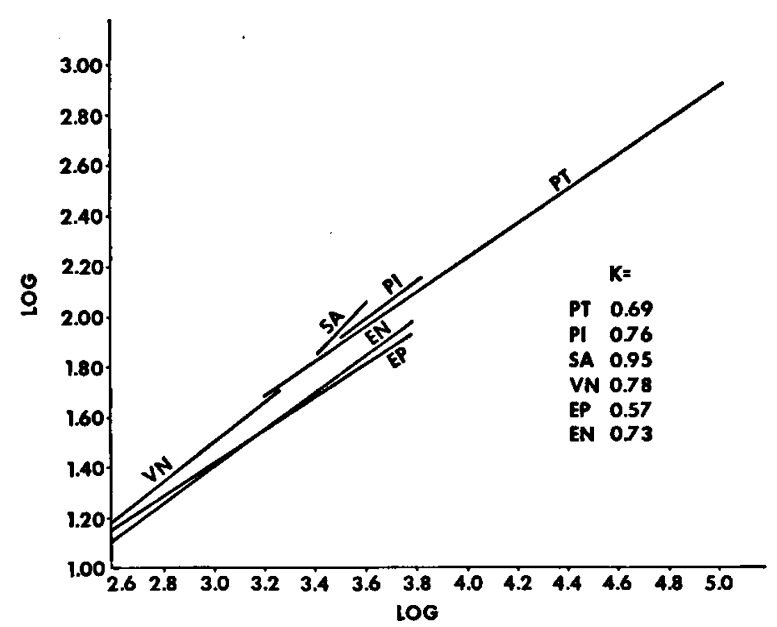

Fig. 2. Régressions des divers groupes selon le régime alimentaire. En ordonnées: poids encéphaliques. En abscisse : poids somatiques. Logarithmes. Les valeurs de $k$ pour chaque équation $\log y=\log b+k \log x$ sont indiquées. Symboles: PT = ptéropidés; PI = piscivores; SA = sanguivores; VN = végétariens du Nouveau Monde; $\mathrm{EP}=$ entomophages paléarctiques; $\mathrm{EN}=$ entomophages du Nouveau Monde.

recourrai au travail déjà cité de Dubois (1914) pour certains chiffres concernant les primates; à celui de Bauchot \& Stephan (1966) pour des valeurs obtenues sur des insectivores et des primates; enfin à une communication additionnelle inédite sur des données recueillies de diverses sources par le Dr. Stephan pour quelques autres primates encore. Les données de tous ces auteurs ont été utilisées conjointement avec les miennes pour le calcul en $\% 0$ des poids relatifs des encéphales. Un résumé est présenté dans la table IV. L'appendice donne le détail des données tirées des auteurs cités. Les poids somatiques sont ceux indiqués dans les publications originales $\mathrm{mo}$ in $\mathrm{s}$ les poids encéphaliques correspondants.

Les trois ordres en cause (chiroptères, insectivores, primates) se prêtent à des comparaisons instructives. Ils sont généralement considérés comme relativement proches l'un de l'autre. Diverses espèces, fossiles ou actuelles, suggèrent cette proximité ou parenté relative. Certains caractères du squelette sont intéressants de ce point de vue (denture en particulier). On a, de longue date, proposé l'hypothèse que leur origine phylétique commune se trouve quelque part chez un ancètre inconnu de type insectivore.

On constate ici que les rapports encéphaliques ou poids relatifs varient, dans chaque ordre, entre des limites très semblables. Les valeurs élevées 43.6 (Galago), 43.2
(Tarsius), 40.4 (Midas), 38.8 (Hapale) et 37.0 (Callithrix) sont assez exceptionnelles. Des 50 espèces de primates mentionnées ici, 45 se situent en dessous de la valeur $30 \%$ en ce qui concerne le rapport, c'est-à-dire dans une zone de valeurs où elles rejoignent les insectivores et les chiroptères. L'homme lui-même n'est pas, si l'on considère ces données immédiates en $\%$, placé particulièrement haut $(21-24 \% \%)$. De plus, dans d'autres ordres où l'on trouve nombre d'espèces comparables dans leur ensemble aux chiroptères, par leur poids somatique, en observe que les poids encéphaliques relatifs se situent dans les mêmes régions c'est-à-dire ne dépassent pas 30\% le cas chez les rongeurs et les carnivores. D'après les données des divers auteurs déjà mentionnés, la grande majorité de ces points se trouvent même en dessous du niveau $20 \%$. C'est le cas pour environ $80 \%$ des rongeurs, $77 \%$ des carnivores, $72 \%$ des insectivores et $65 \%$ des primates. Environ $70 \%$ des chiroptères sont dans la même position. Quant aux ordres dans lesquels la taille somatique est nettement plus considérable, ils se situent plus bas encore, d'ordinaire sous $10 \%$ (cf. les cétacés, lagomorphes, édentés, ongulés et marsupiaux dont les espèces fournissent rarement un poids encéphalique relatif atteignant 11 ou $12 \%$ ).

Ces observations montrent: a) que les rapports encéphaliques chez les mammifères semblent être tous compris entre les chiffres 0 et $45 \%$, cette amplitude de variation étant, pour le moment du moins, suffisante pour tous les besoins adaptatifs de la classe, répondant sans doute aux nécessités générales des contrôles nerveux internes et externes; b) que les valeurs supérieures à $30 \%$ sont rares et que la plupart sont même inférieures à $20 \%$; c) que les primates présentent un certain nombre de valeurs élevées mais qu'ils sont suivis de près en cela par les chiroptères d'abord, par quelques insectivores ensuite puis par certaines petites espèces de carnivores et de rongeurs. Nous sommes ainsi ramenés une fois de plus à l'idée générale de la corrélation négative entre le poids relatif de l'encéphale et le poids absolu du corps (règle de Haller - cf. Pirlot, 1969a). Pour l'examiner plus aisément, on peut, avec les données ci-dessus, dresser le graphique fig. 3 qui présente comparativement les cas des chiroptères, des insectivores et des primates. Parmi les chiroptères, j'ai distingué par des lettres six catégories. Les poids somatiques ont été remplacés par leurs logarithmes de manière que le graphique se réduise à des dimensions commodes. 
Table II. Estimations de corrélation et de régression pour les relations intraspécifiques.

$\mathbf{r}=$ coefficient de corrélation; Signif. $\mathbf{r}=$ signification de $\mathbf{r}$ au niveau $0.05 ;$ N.S. $=$ non significatif; $(S)=$ douteusement significatif; $\mathrm{B}+\mathbf{k X}=\log \mathrm{b}+\mathbf{k} \log \mathbf{x} ; \mathbf{n}=$ nombre de spécimens.

$\begin{array}{lccrr}\text { Spécimens d'Asie } & \text { r } & \text { Signif. r } & \text { B + kX } & \text { n } \\ \text { Cynopterus horsfieldii } & 0.35 & \text { N.S. } & 1.45872+0.16928 \text { X } & 16 \\ \text { C. brachyotis } & 0.80 & \text { N.S. } & 0.86485+0.31120 \text { X } & 5 \\ \text { Eonycteris spelaea } & 0.47 & \text { N.S. } & 1.61236+\mathbf{0 . 1 2 3 6 8 X} & 14 \\ \text { Hipposideros bicolor } & 0.04 & \text { N.S. } & 1.30891+0.02308 \text { X } & 11\end{array}$

Spécimens d'Europe et d'Afrique

$\begin{array}{lcc}\text { Rousettus aegyptiacus } & 0.58 & \text { N.S. } \\ \text { Hipposideros caffer } & 0.46 & \text { (S) } \\ \text { Nycteris arge } & 0.39 & \text { N.S. } \\ \text { Myotis myotis } & -0.05 & \text { N.S. } \\ \text { Rhinolophus hipposideros } & 0.21 & \text { N.S. } \\ \text { Miniopterus schreibersi } & 0.23 & \text { N.S. } \\ \text { Chaerophon leucostigma } & 0.60 & \text { N.S. } \\ \text { C. limbatus } & -0.42 & \text { N.S. }\end{array}$

N.S.

(S)

N.S.

N.S.

N.S.

N.S.

$1.87771+0.12490 X$
$0.96043+0.17361 X$
$1.31123+0.07509 X$
$1.74613-0.03088 X$
$1.31239+0.06006 X$
$1.35808+0.03135 X$
$0.72015+0.25458 X$
$2.16797-0.22370 X$

Tout d'abord, l'existence de la corrélation négative signalée est amplement confirmée par la distribution générale des points figuratifs du diagramme. En outre, on y voit que les ordres de mammifères impliqués se répartissent d'un manière propre à chacun, et cela dépit du fait qu'un certain nombre de poids somatiques absolus sont à peu près les mêmes pour plusieurs espèces appartenant à chacun de ces ordres distincts. On observe, en effet, obliquement et dans la partie inférieure gauche du graphique, la $z$ on e des in sectivores (points - zone III). Nettement au-dessus, s'étend loin vers la droite la z o ne des pri$m$ a te s (croix-zone I); chez ceux-ci, on notera, pour des valeurs données de l'abscisse, les écarts notables auxquels il a déjà été fait allusion, à savoir ceux de l'homme (croix encerclée) et ceux des prosimiens ou petits singes mentionnés (croix soulignées). Quant aux chiroptères, ils se disposent d'une manière remarquable. Les espèces entomophages (lettre E) se mêlent pour la plupart aux insectivores: cette coincidence de localisation des mangeurs d'insectes appartenant à deux ordres distincts mérite de retenir l'attention. Seuls s'écartent un peu de la zone des entomophages certains spécimens à poids encéphalique relatif exceptionnellement élevé. Ils appartiennent à de très petites espèces, généralement de moins de $10 \mathrm{~g}$ de poids somatique: il s'agit donc des formes extrèmes d'un groupe que en comporte d'autres de 15 à 20 fois plus lourdes. Cet effet du nanisme sur l'encéphale est connu dans d'autres ordres: les petits primates à hauts rapports encéphaliques cités ci-dessus en sont sans doute d'autres exemples. Ces chiroptères entomophages miniatures rejoignent ainsi dans le graphique les chiroptères non-entomophages, en majorité des végétariens (lettre $\mathrm{V}$ ) qui se distribuent obliquement dans une zone in termédi a ire (zone II) entre mangeurs d'insectes et primates (ces derniers étant d'ailleurs plus ou moins végétariens ou omnivores selon la conception courante). Parmi les chiroptères non-entomophages, on peut considérer comme animaux très spécialisés les sanguivores (lettre $S$ ) et le piscivore (lettre P) : les premiers se situent particulièrement haut tandis que le second, sans atteindre une valeur extrêmement élevée, se détache néanmoins nettement des entomophages avec lesquels il est pourtant étroitement apparenté (par sa forme cogénérique $N$. labialis). Les lettres $\mathrm{A}, \mathrm{B}$ et $\mathrm{C}$ au sommet du graphique attirent l'attention sur les trois régions ou bandes verticales de relèvement. L'espèce végétarienne pourvue du plus haut rapport (bande B) est aussi une forme très spécialisée : le petit nectarivore Glossophaga (lettre V dans un carré). Par contre, la grande forme nectarivore Leptonycteris (même symbole que le précédent) se situe dans la région non relevée des végétariens de sorte qu'il est possible que la forte encéphalisation de Glossophaga soit due plutôt au nanisme qui affecte cette forme qu'à sa spécialisation éthologique. Toutefois, les nectarivores posent un problème particulier. On ne sait trop dans quelle mesure leur régime est primaire ou secondaire. II est bien connu qu'on trouve assez souvent des insectes dans l'estomac de ces animaux. On pourrait supposer qu'ils ne sont devenus buveurs de nectar qu'à l'occasion d'une habitude prise de 


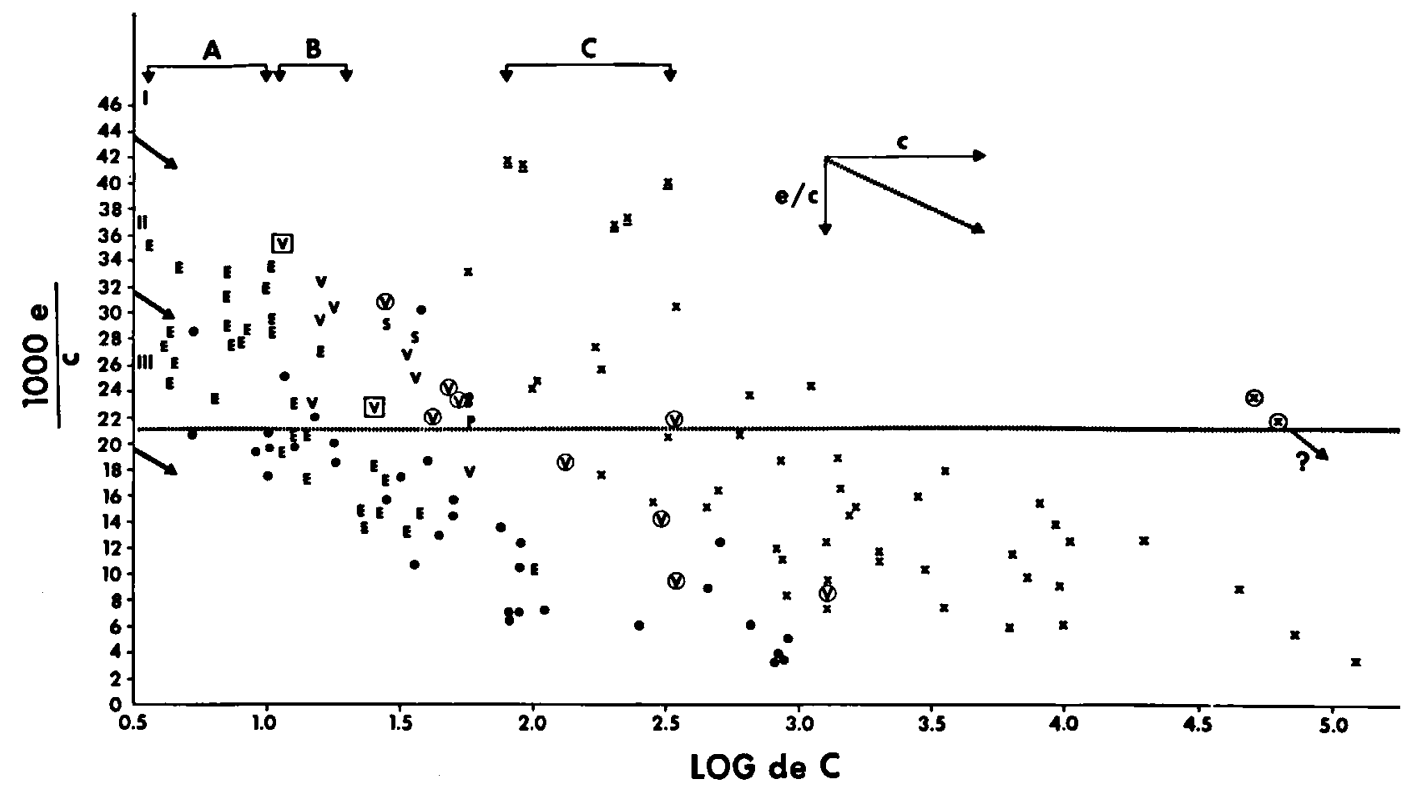

Fig. 3. Graphique du poids encéphalique relatif en ordonnées $\mathrm{y}=(1000 \mathrm{e} / \mathrm{c})$ par rapport au poids absolu du corps en abscisse. Points $=$ insectivores; $\mathrm{E}=$ entomophages; $\mathbf{V}=$ végétariens frugivores d'Amérique tropicale; $\mathrm{V}$ dans un carré $=$ nectarivores; $\mathrm{V}$ encerclés $=$ ptéropidés; $\mathbf{S}=$ sanguivores; $\mathrm{P}=$ piscivores; croix $=$ primates; croix soulignées = Hapale, Midas, Callithrix,

chasser les insectes dans les fleurs, habitude associée elle-même à l'allongement exceptionnel de leur museau et de leur langue. D'après le graphique fig. 3, la position de la grandeur relative de l'encéphale chez les nectarivores ne paraît pas très claire et Leptonycteris est bien près des entomophages. Notons, en passant, que les frugivores d'Asie et d'Afrique (ptéropidés) sont aussi des animaux à museau relativement long et que certains d'entre eux ont également la langue très allongée (Macroglossus).

Dans ce groupe des végétariens de l'Ancien Monde (V dans un cercle), groupe qui comprend de petites et de très grandes espèces, on voit spécialement bien la décroissance du rapport encéphalique avec la croissance du poids somatique. On notera aussi que les chiroptères entomophages de l'Ancien Monde présentent en général des poids encéphaliques relatifs de loin supérieurs à ceux des espèces de même régime d'Amérique du Sud : sur 16 entomophages paléarctiques et paléotropicaux, en effet, 8 se situent au niveau de la valeur maximale (28.6) observée parmi les représentants néo-tropicaux de ce groupe, voire au-dessus de cette valeur. Chose curieuse, il ne semble exister chez les insectivores que peu de relèvements pro-
Galago et Tarsius; croix encerclées $=H$. sapiens. Bande verticale $\mathbf{A}=$ région de relèvement des rapports pour les chiroptères entomophages; bande verticale $\mathrm{B}=$ région de relèvement pour les chiroptères non-entomophages; bande verticale $\mathrm{C}=$ région de relèvement des primates. Autres détails : voir le texte.

noncés du rapport encéphalique : le seul cas vraiment clair est celui de l'espèce spécialisée Scalopus aquaticus à rapport 30.2 pour un poids somatique de $38 \mathrm{~g}$. Les 5 autres indices supérieurs à $20 \%$ chez les insectivores sont moins remarquables.

Ces observations présentent un intérêt particulier si l'on admet que les chiroptères et les primates en général ont pu dériver d'un type insectivore non-spécialisé. Du point de vue de la relation encéphale/corps discutée ici, ces deux évolutions distinctes n'ont pas simplement consisté en des déplacements des points vers la droite et le bas du diagramme mais aussi en des $\mathrm{ch}$ a $\mathrm{n}$ ge m e $\mathrm{nts}$ de nive a ux. En d'autres termes, elles ont impliqué : a) dans une région moyenne, avec un accroissement de taille absolue modéré, un maintien du poids encéphalique relatif à une valeur faible, de niveau insectivore, peut-on dire (moins de $21 \%$ par exemple); b) ou même, avec un accroissement considérable de taille somatique, une baisse de ce poids relatif de l'encéphale, qu'on observe chez les gros primates; c) mais par ailleurs, un relèvement du même poids encéphalique relatif chez les très petites formes de $\mathrm{ch} \mathrm{a} \mathrm{c} \mathrm{u} \mathrm{n}$ de ces deux ordres, jusqu'à des valeurs jamais atteintes par les insectivores de même grosseur 
corporelle (de 22 à $43 \%$ o0 pour des chiroptères et des primates de logarithme somatique inférieur à 3.00). Dans le graphique, la ligne pointillée tirée à la hauteur 21 des ordonnées aide à mieux voir cette répartition des rapports.

Il est peut-être permis d'aller plus loin et de spéculer sur la synchronie ou la diachronie de tels événements évolutionnaires. Faut-il dire que le premier phénomène ou phénomène "primaire" a été l'augmentation générale de la taille somatique avec $m a$ intie $n$ approximatif de la valeur du poids encéphalique relatif tandis que les formes miniatures à cerveau relativement énorme résulteraient d'un nanisme somatique "secondaire" ? Ou bien, au contraire, l'apparition de petites formes à cerveau augmenté est-elle à l'origi n e de ces dérivations tandis que les espèces les plus grosses (somatiquement) en seraient issues s e c o n d a i$\mathrm{r}$ e $\mathrm{m}$ e $\mathrm{nt}$ avec baisse concomittante de la valeur du rapport ? Il faudrait beaucoup de documents paléontologiques pour trancher ce dilemme. Des correspondances morphologiques nombreuses et détaillées entre les très petites formes de chiroptères et de primates d'une part, les insectivores d'autre part indiqueraient peut-être que la seconde hypothèse est préférable. On se souviendra d'ailleurs à ce sujet que, depuis longtemps, on a suggéré que l'homme pourrait n'être qu'un "singe néoténique" du point de vue de son volume cérébral en particulier (mais aussi d'autres points de vue, par exemple de celui de sa pilosité). Cette suggestion peut conduire à une simplification excessive de la présentation des faits mais elle semble pouvoir s'appliquer à un certain nombre de cas dans l'évolution des mammifères, par exemple ici. Dans cette hypothèse, la résultante des deux variations (accroissement du corps, baisse du rapport encéphalique) à partir de formes relativement petites et d'emblée hyperencéphalisées se présenterait comme on l'a symbolisé par un "quadrilatère de forces" sur la figure 3. Cette façon de voir présente l'avantage d'insister sur le fait qu'au point d'amorçage d'une lignée caractérisée par une augmentation générale de taille peut (ou doit ?) se situer une mutation augmentant le nombre de cellules encéphaliques préalablement à la réalisation d'un volume somatique accru. Il y a certainement là une possibilité de bifurcation phylétique importante. De tels accroissements encéphaliques déterminants ont pu se produire plusieurs fois, en divers points et avec diverses intensités, dans un stock ancestral. Ceci pourrait signifier que le groupe des chiroptères est polyphylétique. C'est fort possible mais ce n'est pas nécessairement le cas : un intermédiaire "protochiroptère" commun aux entomophages, frugivores, nectarivores, sanguivores et piscivores a pu exister; il se situerait dans la région "insectivore gauche" du graphique fig. 3 et aurait donné lieu à une radiation adaptative vers la zone chiroptère mais on ne peut le localiser au moyen des données discutées ici.

Si l'on admet que l'amorçage d'une bifurcation phylétique par accroissement encéphalique relatif peut se situer n'importe où dans la zone oblique occupée par un ordre, on trouvera sans étonnement les points figuratifs de l'homme très audessus de l'extrémité droite de la zone primate. C'est là un "départ" auquel on pouvait s'attendre, dans lequel l'homme (néoténique si l'on veut) se détache nettement de ses co-primates de même poids somatique. Avec un encéphale considérablement accru c'est-à-dire avec des cellules cérébrales devenues peut-être très vite extrêmement abondantes, voire provisoirement surabond a n t e s, l'espèce Homo sapiens est susceptible de se développer en une lignée "sapienne" orientée selon la flèche en trait pointillé (fig. 3). Il semble que, de fait, chez certaines races actuelles du moins, le poids somatique se soit accru depuis quelques siècles sans qu'on remarque une hausse correspondante du poids encéphalique : l'homme européen de 1969 serait trop grand pour revêtir les armures du Moyen-Age et la taille moyenne dans les armées occidentales modernes n'a cessé de croître depuis 100 ans tandis que le volume moyen du cerveau n'aurait pas, selon des rapports verbaux, augmenté proportionnellement.

L'idée qu'une divergence phylétique vers des formes plus grosses s'amorce par des mutations (ou soit précédée de mutations) qui accroissent le nombre des cellules encéphaliques me paraît assez importante. A la racine d'une telle bifurcation, en effet, se pose nécessairement le problème pratique d'un contrôle au moins maintenu, sinon amélioré, des relations du soma avec son environment et des parties du soma entre elles. L'hypothèse suggérée ici répond à ce problème. Car, pour un soma en cours d'accroissement phylogénique (de volume et de poids plus grands), un encéphale plus fourni en neurones est très probablement une assurance a priori d'avantage sélectif.

Dans ces remarques, je n'ai voulu que proposer une direction de réflexion; seules des observations nouvelles peuvent confirmer les hypothèses impliquées ici. 


\section{NOTES}

1) Il va de soi qu'on peut aussi supposer qu'au contraire l'accroissement som a tique est primaire et l'augmentation du rapport encéphalique secondaire, ou encore que des mutations synchrones ont assuré pratiquement la simultanéité des deux accroissements, somatique et encéphalique. Cela me paraît toutefois correspondre moins bien à ce que nous savons du jeu normal de la sélection. De nouveau, seuls des documents paléontologiques abondants et peut-être inexistants jusqu'ici permettraient de mieux discuter de ces possibilités.

2) On ne peut pas s'attendre que la corrélation négative entre poids relatif de l'encéphale et poids absolu du corps soit sans exception chez les vertébrés en général et chez les mammifères en particulier. Il est bien connu (et je ne peux entrer ici dans le détail) que la règle de Haller ne s'applique pas dans certaines lignées où l'on a détecté une augmentation du poids encéphalique relatif parallèle à celle du poids somatique : s'il y a des cas où la notion d'évolution progressive semble s'appliquer, ce sont sans doute ceux-là. Mais il n'en reste pas moins vrai que la corrélation négative en cause, associée à la bien connue "règle de Cope" se vérifie dans l'ensemble des vertébrés. Il est probable que certaines des exceptions à la règle de Haller se rattacheraient précisément à un mécanisme d'amorçage phylétique tel que celui suggéré ci-dessus et commenté plus en détail ci-après.

3) L'idée d'une surabondance provisoire de neurones, dans toute évolution générale de l'encéphale qui implique un accroissement de volume, n'est pas acceptée par tout le monde. Les neurones a p p a r m ment inutiles à une étape initiale peuvent, dit-on, être en réalité tout à fait nécessaires pour la croissance générale prolongée, pour l'intensification éventuelle du métabolisme et pour une reproduction plus active. Ceci est possible mais nous demeurons en tout cas dans le domaine de l'hypothèse. Ce qui est, par contre, certain, c'est que le rôle physiologique de masses neuroniques importantes demeure imprécis sinon douteux aux yeux de l'expérimentateur. Et l'on doit aussi admettre que, s'il est un tissu que l'on peut soupçonner de pouvoir exister bien avant de fonctionner pleinement, c'est bien le tissu encéphalique, constitué de tant d'unités discrètes dont le fonctionnement effectif dépend de la mise au point de synapses innombrables, ce qui peut exiger beaucoup de temps.

\section{B. Coefficients intraspécifiques (ontogéniques)}

Comme je l'ai fait dans la première partie de ce travail, je ne considérerai ici que les espèces représentées par au moins 8 spécimens (table II). Du groupe paléarctique, je n'ai disposé que de quatre formes remplissant cette condition. Si l'on y ajoute 9 espèces néo-tropicales (cf première partie, table II), on constate que les 13 valeurs de $k$ intraspécifique se distribuent entre 0.02 et 0.76 . On peut admettre que les valeurs inférieures à 0.10 sont "anormalement" basses : elles sont sans doute dues en partie à l'imprécision de mes mesures sur des spécimens très petits et difficiles à manipuler en campagne (Hipposideros bicolor, récolté en Malaisie); les encéphales étaient très légers par rapport à la sensibilité de ma balance et, tous les chiffres étant presque égaux, la pente pour cette espèce est presque nulle. En excluant ces spécimens par précaution, on obtient une amplitude de variation de 0.12 (Eonycteris) à 0.76 (Noctilio leporinus). Pour 5 espèces d'insectivores malgaches, Bauchot \& Stephan ont trouvé des valeurs variant de 0.18 à 0.36 . On cite d'habitude la moyenne de tels coefficients pour un groupe donné d'espèces, voire pour toute une classe. L'utilité d'une telle moyenne est douteuse. Notons, néanmoins, pour céder à la coutume, que, pour la douzaine d'espèces que représentent ici 8 spécimens au moins, cette moyenne est de 0.26 . Bauchot \& Stephan (1964) donnent 0.23 pour les insectivores malgaches. Dubois (1914) trouvait 0.25 pour diverses espèces de mammifères et Stephan (1954) confirme ce chiffre pour une série de races de chiens domestiques. Wirz (1950), étudiant divers mammifères, a signalé des variations allant de 0.22 à 0.85 . Il semble que la plupart des auteurs n'aient pas trouvé une variabilité aussi considérable que celle observée parmi mes chiroptères. En particulier, le chiffre de 0.76 peut surprendre. Il est pourtant incontestable: il ressort de données brutes obtenues sur place de la manière la plus simple, sur un échantillon homogène et tel qu'aucune confusion avec une autre espèce n'est possible (spécimens d'une grotte des falaises de Point Gourde, Trinidad). Je n'avais, dans cet échantillon, aucun sujet très petit et tous paraissaient adultes (cf. Pirlot, 1969a).

On notera d'autre part que la valeur maximale de $\mathbf{k}$ obtenue chez les chiroptères de l'Ancien Monde est de 0.31 (Cynopterus brachyotis) et que la plupart des autres valeurs dans ce groupe (9 sur 12) sont inférieures à 0.20 . En contraste, la majorité des valeurs intraspécifiques de $\mathbf{k}$ chez les chauves-souris néo-tropicales sont supérieures à 0.20 (10 sur 16) et souvent même supérieures à 0.30 . Or, mon échantillon de l'Ancien Monde contient peu d'espèces végétariennes et beaucoup d'entomophages tandis que l'inverse s'observe dans ma collection sud-américaine. Disposant dès lors les valeurs de $\mathbf{k}$ selon les régimes végétarien et non-végétarien respectivement, on trouve de fait 8 formes sur 13 à valeurs $k>0.20 \mathrm{chez}$ les premiers et 5 formes seulement sur 15 à valeur k $>0.20$ chez les seconds. L'association n'est pas statistiquement significative mais on peut croire que cela est dû à la petitesse des échantillons; elle est fort suggestive et indique que le problème vaudrait d'être réexaminé si l'on disposait de données plus abondantes. 
Table III. Estimations de corrélation et de régression chez tous les frugivores (Ptéropidés) et tous les Entomophages. Même symboles qu'en table II; $\mathrm{S}=$ significatif

Groupes

Ptéropidés

Entomophages $\mathbf{r}$

0.97

0.94
Signif. $\mathbf{r}$

S

$\mathbf{S}$

$$
\begin{gathered}
\text { B }+\mathbf{k X} \\
-0.46924+0.68804 \mathrm{X} \\
-0.31225+0.56726 \mathrm{X}
\end{gathered}
$$

n
Remarque. - L'association signalée ci-dessus d'une part entre les fortes valeurs de $\mathbf{k}$ et les spécimens d'Amérique du Sud, d'autre part entre les faibles valeurs de $k$ et la collection de l'Ancien Monde est, elle, statistiquement significative $(P<0.05)$ mais c'est précisement un exemple où le test n'a qu'un sens mathématique et aucun intérêt biologique: il dépend de la composition de la récolte qui peut être arbitraire, due au choix des sites de capture par exemple.

C. Coefficients interspécifiques (phylogéniques)

Plus intéressante sans doute que le coefficient ontogénique est la valeur in ters pé c if i qu e ou phylogénique de k.

On retrouve d'abord ici le problème de la délimitation du groupe taxonomique à considérer : genre, famille, ordre ou classe? J'ai montré ailleurs qu'en appliquant à des genres voisins la méthode des paires de données de Dubois (1914), méthode qui a fourni pour l'ensemble des mammifères la valeur moyenne $\mathrm{k}=0.56$ que beaucoup continuent à accepter comme valable pour la classe entière, on obtient chez les chiroptères des valeurs de $\mathbf{k}$ extrêmement variables (Pirlot, 1969b).

Une moyenne entre de tels indices $k$ peut $n$ 'avoir aucune signification biologique intéressante. Il en va de même des valeurs de la pente $\mathbf{k}$ de l'équation logarithmique qui ne présente d'intérêt que si l'ensemble du groupe pour lequel on la calcule possède son sens biologique intrinsèque. Ici, les chiroptères ont été groupés selon les régimes alimentaires. Les valeurs de $\mathbf{k}$ sont assez voisines dans les trois groupes néo-tropicaux: végétariens $(0.78)$, entomophages $(0.73)$ et piscivores $(0.76)$, ce dernier groupe étant toutefois monospécifique (k intraspécifique, repris ici à titre de comparaison). Parmi les chiroptères de l'Ancien Monde, on obtient 0.69 pour les ptéropidés et 0.57 pour les entomophages. Stephan (1959) a trouvé $0.71 \mathrm{chez}$ des insectivores: Bauchot \& Stephan (1966) donnent des valeurs variant de 0.613 à $0.680 \mathrm{chez}$ les divers groupes d'insectivores $(0.63$ pour les insectivores de base) et de 0.535 à 0.724 pour quatre lots de prosimiens.

Chez mes ptéropidés, le coefficient est très proche de celui (0.66) que donne Dubois pour quelques comparaisons entre des chiroptères traités exclusivement par paires, valeur qui correspond théoriquement a un accroissement de l'encéphale proportionnel à celui de la surface somatique. Enfin, le coefficient $k$ des vampires est, comme on l'a vu antérieurement, plus élevé que tous les autres (0.95) mais cette famille n'est représentée que par deux espèces de sorte que c'est encore un cas où l'on traite une paire de données (Pirlot, 1969b) mais une paire qui constitue, une entité biologique particulièrement significative.

\section{Conclusion}

Les résultats rapportés dans les deux parties de cette étude ne sont certainement pas simples à interpréter. On peut proposer les conclusions restreintes qui suivent.

1) Loi des surfaces. Pour des groupes clairement définis les uns par rapport aux autres du point de vue éthologique (régimes alimentaires), la "loi des surfaces" caractérisée par la valeur $\mathrm{k}=0.66$ et dont j'ai discuté ailleurs (Pirlot, 1969b) n'est pas observée chez les chiroptères en général. Plusieurs valeurs phylogéniques de $\mathbf{k}$ s'écartent en effet notablement de 0.66 soit en plus soit en moins. Il est cependant remarquable que les ptéropidés aient une valeur très proche de 0.66 ; en fait, sans la présence dans mes données de Pteropus edwarsi, espèce extraordinairement encéphalisée pour cette famille, j'aurais obtenu un coefficient de 0.66 exactement au lieu de 0.69 . Les données trouvées dans la littérature pour les poids somatique et encéphalique de $P$. edwarsi seraient même suspectes si elles ne provenaient apparemment de sources indépendantes.

2) Coefficients $\mathbf{k}$ par groupes. C'est le groupe des entomophages de l'Ancien Monde qui présente, avons-nous vu, le coefficient interspécifique $\mathrm{k}$ le plus bas (0.57). Les coefficients intraspécifiques des espèces de ce groupe sont aussi, on l'a vu, relativement faibles: ils varient de 0.02 à 0.25 alors que ceux des entomophages néo-tropicaux, sans être très élevés, oscillent de 0.14 à 0.53 et que ce groupe sud-américain dans son ensemble présente la valeur interspécifique $k=0.73$. La croissance phylogénique de l'encéphale a donc pu être très différente chez des animaux de même taille moyenne approximative et de même régime (entomophage) mais vivant sur des continents séparés. Ceci n'empêche pas, il faut s'en souvenir, 
Table IV. Amplitude de variation du rapport encéphalique pondérale $(y / x)$ chez trois ordres de mammifères, avec le nom du genre pour chaque extrême

$\begin{array}{lllllll}\text { Chiroptères } & : & \text { de } 8.4 & \text { (Pteropus) } & \text { à } 35.3 & \text { (Glossophaga) } & \text { en } \% \\ \text { Insectivores } & : & \text { de } 3.0 & \text { (Tenrec) } & \text { à } 29.1 & \text { (Sylvisorex) } & \text { en } \% 00 \\ \text { Primates } & : & \text { de } 3.7 & \text { (Gorilla) } & \text { à } 43.6 & \text { (Galago) } & \text { en } \% 00\end{array}$

que ce soit chez certains petits chiroptères d'Europe, d'Afrique et d'Asie qu'on trouve les poids encéphaliques relatifs les plus élevés parmi les mangeurs d'insectes du monde entier (cf. parag. A). Ces valeurs ne sont pas liées.

3. Corrélations et régressions. Les coefficients de corrélation et de régression doivent être considérés avec prudence. Souvent, les nombres de points figuratifs sont petits. Dans le graphique fig. 2 , on observe que les entomophages paléarctiques et les sanguivores se distinguent clairement de tous les autres par leur pente, les premiers ayant une pente faible, les seconds une pente très forte. Quant à l'ordre des grandeurs croissantes des ordonnées à l'origine, soit $\mathrm{B}$, il est le suivant: -1.274 pour les sanguivores, -0.844 pour les végétariens néo-tropicaux, -0.797 pour les entomophages néo-tropicaux, -0.785 pour les piscivores, -0.469 pour les ptéropidés et -0.312 pour les entomophages de l'Ancien Monde.

Bien qu'on ne dispose pas de nombres suffisants pour effectuer des tests satisfaisants de pentes et d'ordonnées à l'origine, il est cependant permis de conclure de ces observations que l'évolution du poids encéphalique a été différente dans les divers groupes de chiroptères considérés et en même temps caractéristique de chacun. Les groupes entomophage et végétarien néo-tropicaux présentent des valeurs de $k$ et de $B$ assez proches les unes des autres. Chose curieuse, ce sont aussi les groupes où il règne encore le plus de confusion taxonomique et éthologique; en particulier, les régimes y sont parfois encore mal définis (fruits et insectes ou nectar et insectes étant acceptés) et la morphologie y est en plusieurs cas très semblable chez des animaux à adaptations éthologiques bien distinctes (phyllostomatidés). On peut se demander si, parmi tous les chiroptères, les formes sud-américaines ne sont pas de différenciation relativement très récente et, en ce cas, pourquoi. Les valeurs de B pour les entomophages et le piscivore du Nouveau-Monde sont aussi fort voisines et certaines des espèces de ces groupes se ressemblent étonnamment (les deux Noctilio). Par contre, si l'on compare les chiroptères néo-tropicaux à ceux de l'Ancien Monde, on observe que les valeurs de $\mathbf{k}$ et de $B$ à la fois sont très distinctes tant pour les entomophages que pour les végétariens. Il y a donc là indication de deux évolutions divergentes d'animaux à régimes alimentaires semblables mais séparés géographiquement depuis peut-être très longtemps. On peut évidemment s'attendre à vcir intervenir dans ces évolutions d'autres facteurs que le conditionnement par la nourriture: il ne s'agit d'ailleurs pas ici de tenter de réduire tout le problème à une simple question de diversification diététique.

4. Encéphalisation et progression. Pour éviter certaines confusions possibles, je n'ai parlé dans cette discussion que de "rapports encéphaliques" et de "poids encéphaliques relatifs" et j'ai peu recouru au terme "encéphalisation" (ou à son équivalent plus ancien "céphalisation") dont le sens ne me paraît pas toujours identique chez tous les auteurs. Néanmoins, si l'on considère séparément les espèces de chiroptères, on pourrait souhaiter les comparer entre elles par un indice d'encéphalisation ou un indice de progression dans l'encéphalisation analogue à ceux qu'on utilisés Bauchot \& Stephan $(1964,1966)$ et Stephan (1967) pour les insectivores et les prosimiens. Mais, dans le cas des insectivores, le fondement de la comparaison se trouvait dans le groupe des insectivores dits de base, définis par Stephan (1967) et par Bauchot \& Stephan (1966). Chez les insectivores, on peut admettre qu'on se trouve en présence de certains types relativement nonspécialisés, sorte de point de départ de la radiation mammalienne. Chez les chiroptères, par contre, toutes les formes sont déjà très spécialisées : ceci est vrai même des entomophages que l'on serait tenté de rapprocher des insectivores sur la base de leur petite taille (dans la plupart des espèces du moins) et de leur communauté de régime. Mais ces chiroptères entomophages n'en sont pas moins des animaux extrêmement spécialisés dans leur morphologie et leur physiologie nerveuse en rapport avec leur mode de vie. Quant à la position prétendument primitive des ptéropidés, en tant que groupe, j'en remets la discussion à un autre travail : ce "primitivisme" d'ensemble est des plus douteux et doit à tout le moins être précisé et nuancé.

On peut naturellement, dans la perspective de l'hypothèse déjà évoquée que les insectivores et particulièrement les insectivores de base actuels 
sont les plus proches de la souche ancestrale commune, effectuer par référence à ces derniers des comparaisons d'espèces appartenant à d'autres ordres. Il serait donc justifié d'étudier comparativement les indices d'encéphalisation ou de progression des chiroptères et des insectivores de base, voire d'autres formes encore en même temps: ceci a été réalisé et sera publié prochainement (Pirlot \& Stephan, 1970).

5. Différenciations structurelles et modes de vie. Il est bien certain, quel que soit l'intérêt de l'étude quantitative des variations encéphaliques, que le problème de l'évolution du système nerveux ne peut pas se réduire à une question de poids cérébral global (Pirlot, 1969a). Une masse encéphalique donnée peut être très diversement structurée et non moins diversement utilisée.

C'est pourquoi l'étape la plus intéressante de l'étude de l'encéphale des chiroptères consiste en recherches sur les différences structuerelles qui peuvent exister entre ces encéphales dont on vient de voir qu'ils ont avec le corps des relations pondérales très variables. Chaque partie de l'encéphale peut varier considérablement en développement relatif par rapport aux autres et par rapport au corps en même temps. On peut s'attendre que les masses corticales et sous-corticales jouent chez les diverses espèces des rôles très variables en relation avec le spécialisation éthologique de chacune. Il serait surprenant qu'il en fût autrement puisque l'encéphale total reflète déjà dans son développement quantitatif une nette relation, directe ou indirecte, avec le régime alimentaire.

L'étude anatomique de détail des chiroptères utilisés dans les deux parties de ce travail est en cours. Les comparaisons qu'elle présentera porteront: a) sur l'importance relative de diverses parties de l'encéphale chez les entomophages, végétariens, sanguivores et piscivores respectivement; b) sur le développement des diverses parties de l'encéphale chez les insectivores d'une part, chez les chiroptères (et éventuellement quelques primates) d'autre part. L'intérêt de cette morphologie comparative de ce qu'on peut appeler un "carrefour de mammifères" est évident de soi, en raison de la diversité des adaptations et des modes de vie des nombreuses espèces impliquées.

Enfin, parmi les espèces de chauves-souris à haut rapport encéphalique, on compte non seulement les plus petites formes mais aussi celles qui sont le mieux pourvues du point de vue du sonar (vespertilionidés, rhinolophidés, hipposidéridés). Mais, au même niveau du rapport encéphalique, se situent aussi certains végétariens et les sanguivores. Par exemple, un ensemble d'espèces appartenant à ces trois groupes se localisent au-dessus du niveau $26 \%$ oo dans la figure 3 . Ces espèces devront être comparées du point de vue de leur structure encéphalique. A première vue, les frugivores semblent avoir plus de cortex que les entomophages, et l'on admet aussi qu'ils sont moins bien équipés en ce qui concerne le sonar. Le problème est évidemment complexe. Des différences structurelles précises sont susceptibles d'être mises en rapport avec l'importance relative, pour chaque espèce en particulier, de chacun des trois sens : ouie, vue, odorat. Les études en cours donnent déjà sur ce sujet des informations intéressantes qui seront publiées ultérieurement. On voit d'ores et déjà que ce problème de la signification biologique générale ou spécialement éthologique de l'architecture interne du cerveau est directement issu de la simple considération des données quantitatives sur l'encéphale total qui viennent d'être commentées dans ce travail d'introduction.

Note. - Au moment où cet article allait être envoyé à l'éditeur, j'ai trouvé le travail remarquable de Rensch (1958) où sont exposées des hypothèses qui rejoignent les idées exprimées ci-dessus. Dans son paragraphe sur les différences allométriques des régions corticales antérieures, chez les vertébrés, Rensch reprend de l'un de ses travaux antérieurs la notion de "postintégration des fonctions" nerveuses, c'est-à-dire de l'acquisition "secondaire" de fonctions nouvelles par des masses nerveuses accrues. Exprimée pour tous les vertébrés, des poissons à l'homme, cette idée correspond certainement à ce qu'on sait de l'émergence graduelle d'un cortex morphologiquement et physiologiquement de plus en plus important. Mais cette hypothèse peut être appliquée à l'encéphale total, d'une part, et en même temps à des cas de bifurcations phylétiques de niveau inférieur à l'embranchement, d'autre part.

On notera, en premier lieu, qu'un accroissement du cerveau antérieur préparatoire à de nouvelles fonctions pourrait en principe s'opérer sans changement dans le poids relatif global, se réalisant aux dépens d'autres parties de l'encéphale. En second lieu, je pense que mes données, qui concernent des cas bien concrets tirés des mammifères, démontrent précisement que l'encéphale total peut être considérablement accru dans certains types que l'on peut considérer comme des "amorçages phylétiques". En outre, il est d'importance qu'on ait trouvé ici de tels cas dans le cadre d'une seule classe ou même d'un seul ordre (voire d'une même famille). Car, tant qu'on ne parle que de l'embranchement des vertébrés (ou des chordés) dans son ensemble, la chose semble aller de soi tant sont évidentes, d'une part, les accroissements relatifs de l'encéphale, d'autre part les perfectionnements fonctionnels - et l'on peut, en gros, rattacher les seconds aux premiers sans préciser davantage et sans prendre beaucoup de risque. Mais des exemples bien circonscrits 
dans les limites d'un groupe de rang taxonomique relativement bas (un genre, par exemple) ont une valeur démonstrative particulièrement forte.

\section{REMERCIEMENTS}

Pour recueillir les données utilisées dans les deux parties de cette étude, j'ai bénéficié de l'assistance de nombreuses personnes à qui je désire exprimer ma gratitude : le doyen et les professeurs A. Pons et J. Homez de la faculté de médecine de la Universidad del Zulia, Maracaïbo; le Dr. E. Gonzalez, vice-recteur, Universidad de Oriente, Cumana; les autorités civiles de l'Ile de Marga- rita, les missionnaires capucins d'Araguaymujo et de Guayo, Delta de l'Orénoque, Vénézuéla ; les professeurs Kelly, Port of Spain, Trinidad; B. Villa, Universidad Autonoma de Mexico; Tirhunaranan et Mohideen, University of Madras et New College, Madras; Lord Medway, University of Malaya, Kuala-Lumpur ; A. Beck, Kuala-Lumpur; T. Kamiya, Université de Tokyo; le Dr H. Stephan, Max-Planck-Institut für Hirnforschung, Francfort/Main. Les graphiques ont été dessinés par madame H. Farid. Les régressions ont été calculées au Centre de Calcul de l'Université de Montréal (prof. J. Beaudot).

Ce travail a été réalisé avec l'aide financière du Conseil National de Recherches du Canada.

\section{APPENDICE}

Proportion de l'encéphale par rapport au corps chez divers insectivores et primates. Certains termes taxonomiques anciens ont été remplacés par d'autres depuis le temps où sont parus des travaux cités ici; je les ai cependant conservés pour faciliter la référence à ces travaux.

\begin{tabular}{|c|}
\hline $\begin{array}{c}\text { Poids } \\
\text { somatique } \\
\text { moyen en } \mathbf{g}\end{array}$ \\
\hline
\end{tabular}

A. D'après des données communiquées par le $\mathrm{Dr} H$. Stephan et d'origines diverses.

$\begin{array}{lcr}\text { Callithrix pennicillata } & 195.2 & 37.0 \\ \text { Saguinus tamarin } & 329.7 & 31.3 \\ \text { Callicebus moloch } & 644.5 & 24.0 \\ \text { Aotus trivirgatus } & 834.0 & 19.2 \\ \text { Pithecia pithecia } & 1073 & 25.1 \\ \text { Cebus apella } & 3026 & 24.4 \\ \text { Ateles geoffroyi } & 7892 & 13.7 \\ \text { Cercopithecus ascanius } & 3535 & 18.2 \\ \text { Cercopithecus mitis } & 6424 & 11.8 \\ \text { Colobus polykomos } & 9310 & 9.7 \\ \text { Papio cynocephalus } & 18805 & 10.3 \\ \text { Symphalangus syndactylus } & 10370 & 12.4 \\ \text { Pan troglodytes } & 45580 & 9.2 \\ \text { Gorilla gorilla } & 124535 & 3.7\end{array}$

B. D'après des données de Dubois (1914 - avec citations de plusieurs autres auteurs) et de Bauchot \& Stephan (1966).

1. Insectivores

Crocidura (Sorex) araneus

$8.6 \quad 19.7$

Crocidura schweizeri (giffardi)

81.6

6.7

*) Ces données sont tirées de Bauchot \& Stephan et sont présentées par ces auteurs comme "moyennes retenues". Elles incluent, en fait, les valeurs relatives aux mêmes espèces et déjà citées au début de la liste des insectivores. Mais elles en incluent aussi bon nombre d'autres et pour cette raison je n'ai pas fait de correction: il s'agit d'estimations très approximatives et des corrections n'auraient eu aucun effet significatif.
Talpa europaea

Erinaceus europaeus

Solenodon paradoxus

Tenrec ecaudatus

Setifer setosus

Hemicentetes semispinosus

Echinops telfairi

Oryzoryctes talpoides

Nesogale dobsoni

Nesogale talazaci

Limnogale mergulus

Potamogale velox

Erinaceus europaeus *)

Sorex minutus

Sorex araneus *)

Blarina brevicaudata

Neomys fodiens

Sylvisorex megalura

Sylvisorex lunaris

Suncus murinus

Crocidura hildergardae

Crocidura russula

Crocidura niobe

Crocidura jacksoni

Galemys pyrenaicus

Desmana moschata

Talpa europaea *)

Scalopus aquaticus

Chrysochloris asiatica

Chrysochloris stuhlmanni

Elephantulus fuscipes

Rhynchocyon stuhlmanni
Crocidura occidentalis

\begin{tabular}{c|c} 
Poids & $\begin{array}{c}\text { Poids } \\
\text { somatique } \\
\text { moyeéphalique }\end{array}$ \\
relatif en $\%$
\end{tabular}

$\begin{array}{rr}86.5 & 10.9 \\ 881.9 & 3.7 \\ 895.3 & 5.2 \\ 829.4 & 3.0 \\ 246.5 & 6.1 \\ 109.2 & 7.6 \\ 86.9 & 7.1 \\ 43.6 & 13.5 \\ 32.0 & 17.5 \\ 49.6 & 16.0 \\ 90.9 & 12.6 \\ 655.9 & 6.2 \\ 856.7 & 3.9 \\ 5.2 & 21.1 \\ 10.1 & 19.8 \\ 18.1 & 20.8 \\ 14.9 & 22.0 \\ 5.2 & 28.8 \\ 18.2 & 18.6 \\ 35.1 & 10.8 \\ 10.4 & 21.1 \\ 10.8 & 17.6 \\ 11.2 & 25.0 \\ 12.4 & 20.1 \\ 27.6 & 16.0 \\ 56.2 & 23.6 \\ 436.0 & 9.1 \\ 75.0 & 13.4 \\ 38.4 & 30.2 \\ 48.3 & 14.5 \\ 39.1 & 18.9 \\ 55.7 & 23.9 \\ 483.9 & 12.6\end{array}$


2. Primates

Homo sapiens

Homo sapiens

Simia satyrus

Hylobates syndactylus

Semnopithecus maurus

Macacus cynomolagus

Hapale jacchus

Midas rosalia

Nycticebus tardigradus

Tupaia javanica

Tupaia javanica

Tupaia glis

Urogale everetti

Cheirogaleus medius

Cheirogaleus major

Microcebus murinus

Hapalemur sinus

Lepilemur ruficaudatus

Lemur fulvus

\begin{tabular}{c|c}
$\begin{array}{c}\text { Poids } \\
\text { somatique } \\
\text { moyen en } 8\end{array}$ & $\begin{array}{c}\text { Poids } \\
\text { encéphalique } \\
\text { relatif en } \%\end{array}$
\end{tabular}

$\begin{array}{rr}61635 & 20.7 \\ 50935 & 24.2 \\ 73100 & 5.5 \\ 9370 & 13.8 \\ 7328 & 9.8 \\ 11929 & 6.0 \\ 206 & 38.8 \\ 332 & 40.4 \\ 491.8 & 16.6 \\ 101.5 & 25.0 \\ 102.5 & 24.9 \\ 146.9 & 21.5 \\ 270.7 & 15.8 \\ 173.9 & 18.0 \\ 443.2 & 15.3 \\ 52.2 & 34.0 \\ 1290.5 & 7.4 \\ 907.4 & 8.4 \\ 1376.7 & 16.9\end{array}$

\begin{tabular}{c|} 
Poids \\
somatique \\
moyen en $\mathbf{g}$
\end{tabular}

Poids encéphalique relatif en $\%$

Lemur albifrons

Lemur rufiventer

1517.7

14.7

Lemur mongoz

1373.4

1575.9

1938.0

Lemur macaco

2016.5

2968.5

Lemur variegatus

Avahi laniger occidentalis

2968.5

850.3

1258.6

3453.3

Propithecus verreauxi coquereli

Indri indri

6211.7

2754.9

315.4

Loris tardigradus

Nycticebus coucang

Perodicticus potto

Galago demidovi

Galago senegalensis

Galago crassicaudatus

Tarsius syrichta

Tarsius spectrum
587.5

1136.0

77.6

181.2

839.7

83.9

165.4
11.3

11.6

10.6

11.4

9.1

7.7

6.1

16.4

20.9

21.3

12.3

43.6

26.5

12.2

43.2

28.1

\section{BIBLIOGRAPHIE}

Bauchot, R. et H. Stephan, 1964. Le poids encéphalique chez les Insectivores malgaches. Acta Zool., 45 : 63.

— \& -, 1966. Données nouvelles sur l'encéphalisation des Insectivores et les Prosimiens. Mammalia, 30 (1) : 160.

DúoIs, E., 1914. Die gesetzmässige Beziehung von Hirnmasse zur Körpergrösse bei den Wirbeltiere. $Z$. Morph. Anthr., 18 : 323.

Mangold-WiRz, K., 1966. Cerebralisation und Ontogenesis bei Eutherien. Acta anat., 63 : 449.

Pirlot, P., 1969a. Relations pondérales entre l'encéphale et le corps chez les chiroptères. I. Espèces néo-tropicales. Rev. Can. Biol., 28 (2) : 127.

—, 1969b. Le coefficient phylogénique de Snell-Dubois chez les chiroptères. Biom. Prax., 10 (1) : 22.

Pirlot, P. \& H. Stephan, 1970. Encephalization in Chiroptera, Canad. J. Zool., 48 (3) : 433.

RensCH, B., 1958. Die Abhängigkeit der Struktur und der
Leistungen tierischer Gehirne von ihrer Grösse. Naturwissenschaften, 45 : 145, 175.

Stephan, H., 1954. Die Anwendung der Snell'schen Formel $h=k^{s} p$ auf die Hirn-Körpergewichtsbeziehungen bei verschiedenen Hundrassen. Zool. Anz., 153 : 15 .

,- 1959 . Vergleichend-anatomische Untersuchungen an Insectivorengehirnen. III - Hirn-Körpergewichtsbeziehungen. Morph. J. Jb., 99 : 853.

-, 1967. Zur Entwicklungshöhe der Insektivoren nach Merkmalen des Gehirns und die Definition der "Basalen Insektivoren". Zool. Anz. 179 : 177.

WARNCKE, P., 1908. Mitteilung neuer Gehirn- und Körpergewichtsbeziehungen bei Säugern, nebst Zusammenstellung der gesamten bisher beobachteten absoluten und relativen Gehirngewichte bei den verschiedenen Spezies. J. Psychol. Neurol., 13 : 355.

WIRZ, K., 1950. Zur quantitativen Bestimmung der Rangordnung bei Säugetieren. Acta anat., $9: 134$.

Reçu : le 20 mars 1970. 